\title{
QoE Enhancement in Next Generation Wireless Ecosystems: A Machine Learning Approach
}

\author{
Eva Ibarrola \\ University of the Basque Country \\ Mark Davis \\ Technological University Dublin, mark.davis@tudublin.ie \\ Camille Voisin \\ OptiWi-Fi Ltd.
}

See next page for additional authors

Follow this and additional works at: https://arrow.tudublin.ie/engscheleart2

Part of the Systems and Communications Commons

\section{Recommended Citation}

E. Ibarrola, M. Davis, C. Voisin, C. Close and L. Cristobo, "QoE Enhancement in Next Generation Wireless Ecosystems: A Machine Learning Approach," in IEEE Communications Standards Magazine, vol. 3, no. 3, pp. 63-70, September 2019, doi: 10.1109/MCOMSTD.001.1900001.

This Article is brought to you for free and open access by the School of Electrical and Electronic Engineering at ARROW@TU Dublin. It has been accepted for inclusion in Articles by an authorized administrator of ARROW@TU Dublin. For more information, please contact arrow.admin@tudublin.ie, aisling.coyne@tudublin.ie, gerard.connolly@tudublin.ie.

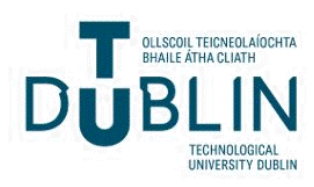




\section{Authors}

Eva Ibarrola, Mark Davis, Camille Voisin, Ciara Close, and Leire Cristobo

This article is available at ARROW@TU Dublin: https://arrow.tudublin.ie/engscheleart2/270 


\title{
QOE ENHANCEMENT IN NEXT GENERATION WiRELESS ECOSYSTEMS: A MACHINE LEARNING APPROACH
}

\author{
Eva Ibarrola, Mark Davis, Camille Voisin, Ciara Close, and Leire Cristobo
}

\begin{abstract}
Next-generation wireless ecosystems are expected to comprise heterogeneous technologies and diverse deployment scenarios. Ensuring quality of service (QoS) will be one of the major challenges on account of a variety of factors that are beyond the control of network and service providers in these environments. In this context, ITU-T is working on defining new Recommendations related to QoS and users' quality of experience (QoE) for the 5G era. Considering the new ITU-T QoS framework, we propose a methodology to develop a global QoS management model for next generation wireless ecosystems taking advantage of big data and machine learning (ML). The methodological approach is based on the use of supervised and unsupervised ML techniques in order to identify both the KQIs relevant for the users and the network performance (NP) anomalies. The proposed methodology links the NP and QoE via inductive ML algorithms and provides information about the areas where corrective actions are required. The results from a case study conducted to validate the model in real-world Wi-Fi deployment scenarios are also presented.
\end{abstract}

\section{INTRODUCTION}

The evolution of Internet users' behavior in recent years, along with the increasing variety of applications and services, has led to Internet access becoming something indispensable for daily life. For that reason, users are becoming more and more demanding in terms of Internet coverage, accessibility, and mobility, leading Internet service providers (ISPs) to consider alternative business models to fulfill these needs. As a result, some of the technologies that were originally considered for providing local Internet access have now emerged as ubiquitous access technologies, leading to complex scenarios where fulfilling the required quality of experience (QoE) has become a significant challenge.

Wi-Fi technology, defined in the IEEE 802.11 standard, is a good example of this. While originally designed to be a wireless local area network (WLAN) technology, today's large deployment of Wi-Fi networks, together with the expected improvements from the $802.11 \mathrm{ax}$ version ( $\mathrm{Wi}-\mathrm{Fi}$ 6 ), has encouraged ISPs to consider new busi- ness models employing this technology as a "ubiquitous access technology for mobile users." Wi-Fi 6 has been developed almost in parallel to the fifth generation (5G), and even though they could be considered competitive technologies, many operators and vendors have envisioned the potential of them working together. The convergence of Wi-Fi 6 and 5G will provide complementary broadband access ensuring the coverage, mobility, and accessibility demanded by users. As a result, many scientific and industrial researchers now envisage 5G as an agnostic radio access network (RAN) comprising multiple wireless technologies. Therefore, even though there is still no clear consensus about what the next generation wireless (NGW) era will embrace, there seems to be a general agreement that it will comprise heterogeneous networks (HetNets) cooperating to maintain the user's QoE. Furthermore, the adoption of new business models to integrate all the capabilities that next generation wireless systems may offer will be crucial. Nonetheless, delivering the required QoE in these complex scenarios will become a major issue.

The International Telecommunication Union's (ITU's) standardization expert group for future networks (SG-13) has been working toward the definition of 5G systems and the development of new Recommendations related to QoS and QoE in the NGW environment [1,2]. Furthermore, in responding to the challenges of managing next generation wireless networks, new groups, like the Focus Group on Machine Learning for Future Networks Including 5G [3], have been established. ITU-T SG-12 has also focused on updating and defining new Recommendations related to QoS and QoE for adapting to the new NGW scenario $[4,5]$. Nevertheless, there is still a need for methodologies that will take advantage of new techniques and mechanisms, such as machine learning (ML) algorithms, to support the deployment of QoS management models as defined in the standardized QoS frameworks.

In this article, a methodology for the implementation of a global QoS management model in NGW ecosystems is proposed. The model takes into account the ITU-T requirements for ML-based QoS assurance for the IMT-2020 network [2], and the methodology aims to include all the aspects that the 5G era will require. Enhancing the QoE 
The challenge when

deploying a Q0S manage-

ment model is to embrace

all the different Q0S relat-

ed aspects (e.g. NP, QoS,

Q0E) and, more important-

ly, quantifying the relation-

ships between them. This

may become a difficult

task when dealing with

next generation wireless

ecosystems, where many

unpredictable factors may

have an influence on the

user's experience. and the satisfaction of the users through new business models to fulfill their requirements are the main targets of the methodology. The identification of the optimal key performance indicators (KPIs) and key quality indicators (KQIs) are essential to achieve this goal.

The proposed methodology represents a major challenge because of the unpredictable nature of the various scenarios considered. With different networks sharing spectrum, for example, operating in the unlicensed industrial, scientific, and medical (ISM) bands, and a response totally dependent on the users' behavior, there are too many factors beyond the control of the network and service provider. Addressing this challenge through ML constitutes the novelty of this proposal. ML techniques can be useful for inferring rules from big data analysis and to identify the KPIs/ KQIs that may lead to an automatic estimation of the quality as perceived by users based on the QoE influence factors. The development of a suitable learning algorithm will be critical to obtaining reliable results.

The remainder of the article is organized as follows. We summarize related work on $\mathrm{ML}$ approaches for QoS management in wireless scenarios. We describe the QoS management model that is adopted and define the QoS-QoE-QoBiz relations to be considered. The proposed methodology to implement the model in NGW scenarios through ML mechanisms is described. We illustrate the experiment carried out to validate the proposed methodology, and finally, we present some conclusions and final remarks.

\section{RELATED WORK}

Many studies and standardization efforts related to QoS and QoE have been conducted in recent years. Most of these show that the assessment of QoS has moved away from network performance (NP) in favor of QoE that is related to the subjective perception of end users. However, as stated in ITU - Telecommunications Standardization Sector (ITU-T) Recommendation G.1000 [6], the challenge when deploying a QoS management model is to embrace all the different QoS related aspects (e.g., NP, QoS, QoE) and, more importantly, quantifying the relationships between them. This may become a difficult task when dealing with next generation wireless ecosystems, where many unpredictable factors may have an influence on the user's experience. In view of this, some recent studies $[7,8]$ suggest using big data analysis and $\mathrm{ML}$ algorithms to model the QoE-QoS relationship.

\section{QoE Models And Machine LeARning}

There is limited research on the modeling of QoE with ML for next generation mobile scenarios. Nevertheless, there are some recent studies focused on analyzing the data captured from the network and users' surveys to model and enhance the QoE [7-9]. The framework presented in [7] is an advance on the other works. In this approach, the process of estimating/predicting the $\mathrm{QoE}$ based on the data collected from the mobile network is described. The state of the art included in this work covers a large set of experiments in several scenarios and for different applications and services in relation to modeling QoE with $\mathrm{ML}$ algorithms. This study also includes a review and analysis of the various factors influencing QoE. Following on the analysis, the authors concluded that three QoE dimensions (i.e., human, system, and context) should be considered when modeling QoE.

Aroussi and Mellouk present a useful survey of ML-based QoE-QoS correlation models in [8]. They suggest that supervised or semi-supervised learning models provide a better fit for the QoEQoS correlation modeling than non-supervised ML. Selecting the most suitable ML technique may be crucial to achieve reliable results, and many algorithms have been proposed in the literature for the analysis and management of the QoS. Jiang et al. describe the different ML algorithms and their application in the 5G environment in [9]. The authors of this article also remark on the importance of choosing the appropriate $M L$ type since the goal of ML is to predict the output of an input by turning observational data into a model that can be used for this prediction. Therefore, depending on the nature of the observational data, different types of learning can be distinguished:

- In supervised learning, each input value of the observational data is given with the corresponding output to form a training set. This set is used to establish a predictive function. Supervised learning is useful for classification and for regression problems.

- In unsupervised learning, only the input values are included in the observational data. The most common application of unsupervised learning is cluster analysis, which is used to find similarities between the input values and extract hidden patterns to group them into clusters.

- Reinforcement learning is based on dynamic iterative learning and decision making processes. The learner is not told which actions to take but instead must determine those that yield the output closest to the target by successive trials.

\section{STANDARDiZATION EFFORTS: Key to 5G}

Standardization bodies also envision the importance of defining new standards on QoS and ML for NGW ecosystems. In January 2018, a workshop on Machine Learning for 5G and Beyond was held in Geneva, Switzerland, in the context of the first meeting of the recently launched ITU Focus Group on Machine Learning for Future Networks Including 5G [3]. Three different Working Groups (WGs) were established:

- WG1: Use cases, services, and requirements

- WG2: Data formats and ML technologies

- WG3: ML-aware network architecture

In this context, ITU-T SG-13 has developed several Recommendations [2, 10] for the specification of an ML-based QoS assurance model for the IMT2020 network. The proposed model (Fig. 1) is organized in three different planes: control, user, and management planes. The control plane includes QoS data collection from the user equipment (UE), access network $(\mathrm{AN})$, core network $(\mathrm{CN})$, and the relevant KPIs. Based on the data pre-processing, modeling and training the QoS anomaly detection/prediction function are developed. The QoS decision results arrive at the management plane 


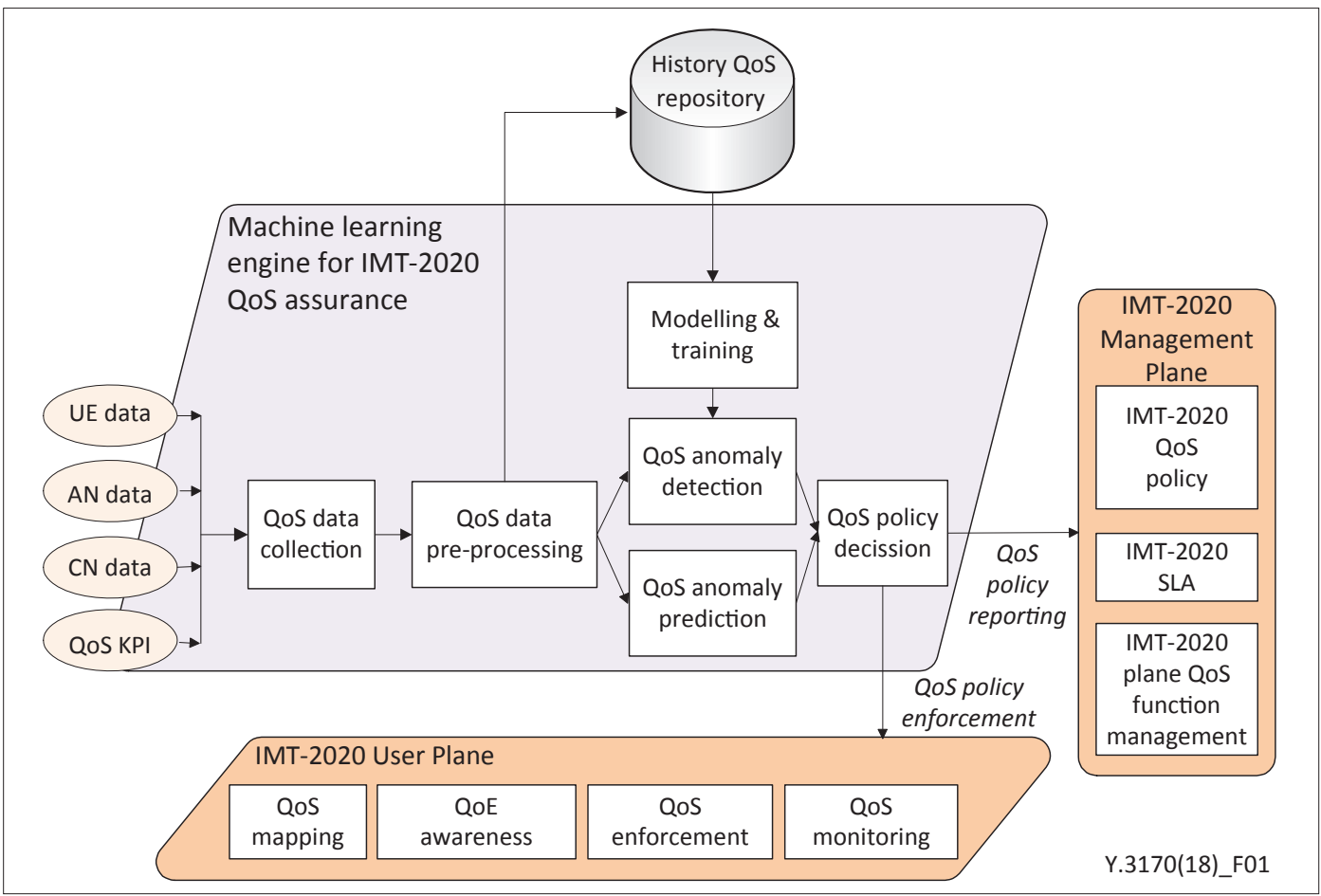

Gathering and collecting data to feed the learning algorithms is essential to infer rules to be applied for both the identification of all the key indicators and the specification of the relationships between the QoS aspects in the different layers of the model.

FIGURE 1. ITU functional model of ML-based QoS assurance [2, 10].

for the QoS assurance management. Finally, the QoS enforcement policy is sent to the user plane to guarantee the user's QoE.

In addition, ITU-T SG-12, the expert group responsible for the QoS/QoE standards, has been active in the editing and updating of Recommendations related to the new mobile scenarios [4, 5]. The Fifth Generation Public-Private Partnership (5G-PPP) is also working actively to define architectures for NGW systems. The work developed by the European Telecommunications Standards Institute (ETSI) Experiential Networked Intelligence Industry Specification Group (ENI ISG) is also active in this area. The ETSI ENI ISG focuses on improving the operator's and user's experience through big data and artificial intelligence by helping in QoE assurance through an "observeorient-decide-act" control loop model.

In spite of the significant advances in the definition of the $5 \mathrm{G}$ ecosystem and the identification of new QoE models and ML techniques for this environment, there is still a need for global QoS management models to be deployed in real-world NGW scenarios. In the following sections, an approach to solving this shortcoming is presented.

\section{QOS MANAgEMENT ModeL: QOXPHERE}

The QoS management model, QoXphere [12], has been adapted from its original architecture to include the new NGW ecosystem requirements. Nevertheless, the basic principles of the model remain; it still takes into account the four viewpoints of QoS of ITU-T G.1000 [6] together with the QoS aspects for mobile networks, as defined in ITU-T E.804 [4]. In this Recommendation (Fig. 2) both QoS technical aspects (NP, KPI, etc.) and non-technical QoS influence factors (customer care, users' expectations, etc.) are considered.
Consequently, the QoXphere model is still organized in four different layers (Fig. 3), although some of the layers have been updated to fit with the new 5G QoS standardized framework.

Figure 4 illustrates the relationships between the different QoS aspects considered in the QoXphere management model. The intrinsic QoS layer still identifies the KPIs to be used for the evaluation of objective QoS. Based on the specified class of service (CoS), the key performance parameters (KPPs) that contribute to each KPI must be identified to evaluate the NP. The results of the first layer analysis feed into the second layer of the QoXphere, where the QoS as perceived by the users (QoP) is estimated. This layer continues to consider the four viewpoints of QoS under ITU-T G.1000. The identification of the KQIs of interest for the users is still the crucial challenge at this stage of the model. The third layer of the model focuses on the evaluation of the assessed QoS. The user's satisfaction is modeled through the QoE information provided by the second layer and the information about the user's expectations. The user's satisfaction values lead to identifying the key risk indicators (KRIs) to estimate the churn probability and establish the key business objectives (KBOs) that constitute the core of the upper layer of the model and analyzes the QoBiz in terms of the profitability of the business models.

This analysis can support "operational efficiency" actions, like defining new advertising procedures, and new billing rates associated with new $\mathrm{KQI} / \mathrm{KPI}$ objectives that will be reflected in the service level agreement (SLA). The new SLA will take into account the updated user's expectations and requirements (through the required $\mathrm{KQ} I \mathrm{~s}$ ) that, at the same time, will aid the identification of the required KPIs to be considered and measured. 


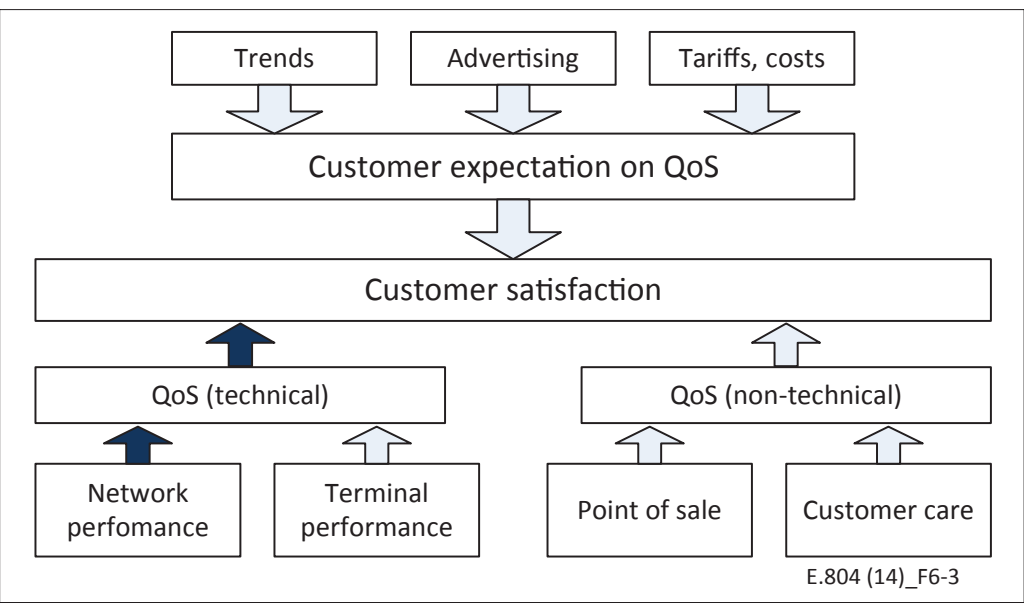

FIGURE 2. QoS aspects for popular services in mobile networks [4].

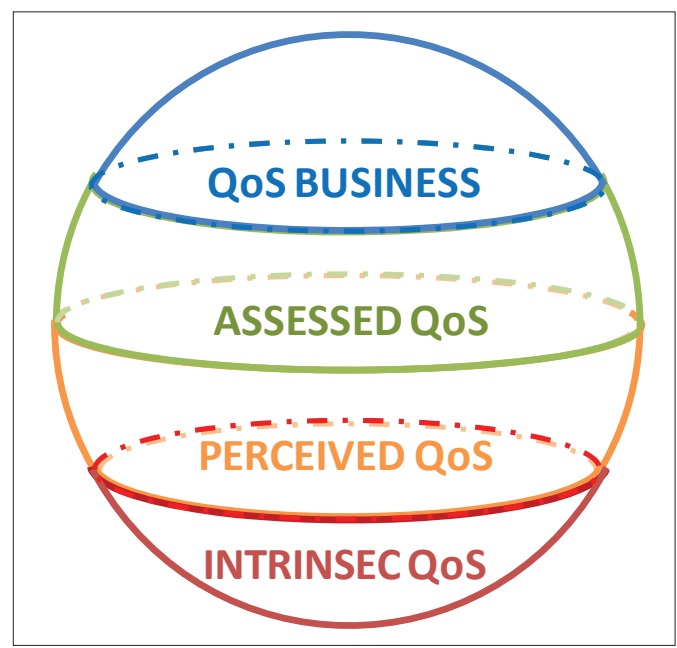

FIGURE 3. QoXphere management model.

\section{MACHINE LEARNING METHODOLOGY}

The methodology proposed in Fig. 5 is based on previous implementations of the QoXphere management model [12], which has been enhanced for deployment in NGW ecosystems. Due to the complex and heterogeneous nature of NGW scenarios, ML mechanisms are proposed to identify some of the QoS aspects and to formulate some of the relations between them. Gathering and collecting data to feed the learning algorithms is essential to infer rules to be applied both for the identification of all the key indicators and the specification of the relationships between the QoS aspects in the different layers of the model.

In addition, the methodology will provide the procedures to continue feeding the model and find the gaps and "hot points" (indicated by red stars in Fig. 5) where intervention is required to improve the QoS and fulfill the QoE requirements. The ITU-T SG-13 QoS framework and ML-based QoS assurance, as proposed in [2, 10] (Fig. 1), have been taken into account when defining the methodology, together with the ITU-T SG-12 principles of managing QoS $[6,11]$.

As stated in ITU-T Recommendation E.802 [11], for any QoS management model to be successful, it is crucial that the identification of the QoS criteria be relevant to the users (based on their requirements/expectations). Nevertheless, many different QoE dimensions and the factors that influence them may have an impact on the user's QoS requirements and expectations. In addition, in the 5G wireless scenario, the network's response will be dependent on the users' behavior and the users' environment. Hence, many contextual and non-contextual agents, not controlled by the providers, may have a significant impact on the users' final satisfaction. For this reason, the first step proposed in the methodology is to understand the users' behavior in each of the scenarios to identify their requirements/expectations and determine the relevant QoS criteria and KQIs. Therefore, since this first step is critical for the QoS management model to succeed, a combination of both contextual and non-contextual information is to be gathered through big data analysis (i.e., step \#1 in Fig. 5). Unsupervised ML techniques (clustering) are proposed for inferring the different scenarios/profiles (the context extraction in Fig. 5) and for finding a user's context influence factors (e.g., location, type of scenario, time of day of usage) and the system influence factors (device type, operating system, etc.). In addition, inductive supervised learning is suggested to infer the rules to identify the QoS criteria and KQI relevant for the users (step $\# 2$ in Fig. 5). In this way, the complex procedure of surveying can be avoided except for the initial training period for the ML model when the user's human influence factors (age, gender, expertise, emotions, etc.) together with their QoS requirements and expectations are captured. Thus, the survey results will provide the particular cases of observation to draw the general rules predicted from a training set drawn from the context, system, and human influence factors. In addition, the subjective information about the user's requirements and expectations are also gathered from surveys (step \#3 in Fig. 5). Once the KPIs relevant to the users have been determined (step \#4 in Fig. 5), the related KPPs will be identified for NP measurements. At this stage, once the KPPs have been identified, the control of the "intrinsic QoS" may start through the QoS data collection (step \#5 in Fig.5). It is recommended to use unsupervised ML techniques to infer suitable radio/channel selection and to detect faults and anomalies in the network behavior from the NP-gathered data. This constitutes the first intervention point where corrective actions may be implemented to enhance the QoS (indicated by the red star A in Fig. 5).

For the study of the QoE (User Plane in Fig. 5 ), the proposed method also considers using both the objective and subjective gathered data. Supervised ML algorithms are suggested (regression model) for the correlation of the NP/QoE [8] using the survey's results (capturing at an initial stage the users' QoE and satisfaction with the service) and the NP collected data, the correlation function between the QoE and NP can be deduced (step \#6 and NP/QoE correlation model in Fig. 5). The context and non-context influence factors will also be crucial when analyzing the user's QoE, and for this reason, they again feed into the training set to learn the rules that will provide the predicted QoE. The ML will 
avoid repeating the surveying process except for the requirement to capture the necessary observation data that will feed the training set process. This training set will be used to deduce the rules that will control the NP/QoE correlation model. Based on the QoE results, the customer satisfaction model (CSAT) described in [12] will estimate the satisfaction of the user with the service. This constitutes the second "hot point" (red star B) where corrective actions may be necessary based on the detected KRIs that may lead to churn and affect the business model.

Finally, the QoS policy decision is made in the management plane. The policy decision will be made based on the QoS anomaly detection/ prediction results and the user's QoE. Furthermore, because the enforcement of the QoS decision policies are required to be supported on the user plane (SLA), the key business indicator (KBO) will be updated on a regular basis (step $\# 7$ in Fig. 5). As referred to in [13], the KBOs are derived from the business areas that are determined to be important for each company, and they should be adjusted through operational efficiency to increase revenue, reduce cost, and improve customer experience. This constitutes the last intervention point where corrective actions may be required (red star D). Billing, advertising, fitting the QoS requirements, and other measures should be analyzed to update the SLA (step \#8 in Fig. 5) taking into account the outputs of the user plane and the control plane.

To conclude, we believe that the proposed methodology can fully accomplish the requirements for ML-based QoS assurance of ITU-T Y.3170 [2].

\section{CASE STUDY: IEEE 802.11 ScENARIOS}

In previous sections we have introduced the principles of the QoE enhancement methodology approach. In this section, we present the implementation of the proposal in a real next generation wireless scenario involving Wi-Fi networks.

\section{Wi-Fi TechNology}

As stated before, Wi-Fi technology is now being considered as one of the key access technologies in the 5G HetNet ecosystem. Nevertheless, implementing QoS management in this type of "unlicensed radio spectrum" wireless network is likely to be complex, especially when users' demands in terms of QoE are continually increasing.

The IEEE 802.11e amendment (2005) was developed to offer new QoS capabilities to the IEEE 802.11 WLAN networks. Under this amendment, new QoS mechanisms, such as the enhanced distributed channel access (EDCA) mechanism, were introduced to Wi-Fi technology at the media access control (MAC) layer, enabling different classes of access categories (ACs) in order to support the prioritization of distinct classes of services. This protocol has been extensively studied, but rarely implemented in practice. The reason is that $\mathrm{Wi}-\mathrm{Fi}$ QoS management is completely dependent on the behavior of users, the coexistence of networks, and many other aspects that the provider cannot control. Consequently, most Wi-Fi service providers have instead resorted to "over-dimensioning" in the deployment of their access points (APs).

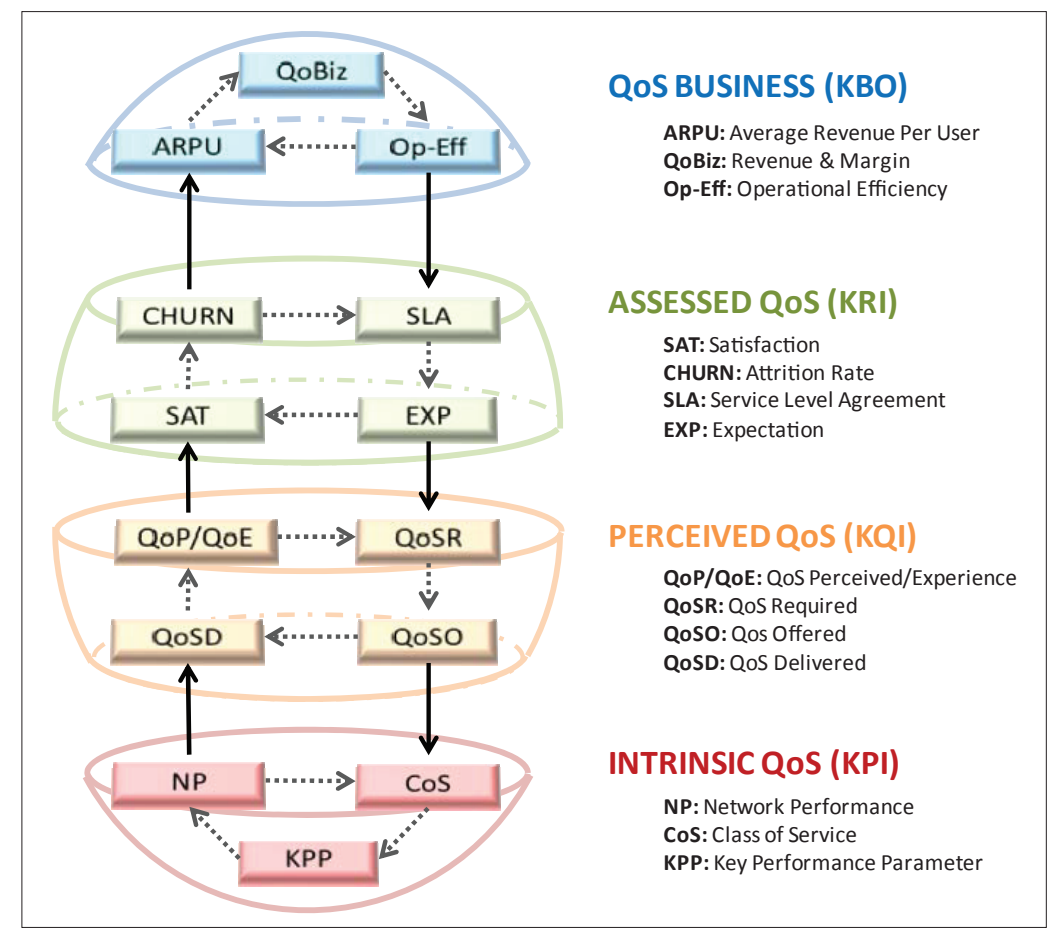

FIGURE 4. QoXPhere: QoS aspects and relationships.

For these reasons, the $\mathrm{Wi}-\mathrm{Fi}$ RAN has been selected to validate the proposed methodology to enhance QoE.

\section{UNSUPERVISED ML:}

\section{Wi-Fi Patterns AND ScEnarios}

For the identification of the different scenarios to be considered, Density-Based Spatial Clustering of Applications with Noise (DBSCAN) clustering ML techniques were used. Comparisons with other algorithms, such as $K$-means, have been carried out, but DBSCAN has achieved better results.

As a first step, ML algorithms were applied only to the most relevant parameters from the extensive dataset obtained from the Wi-Fi probes (bandwidth consumed, transmission rate, number of data frames, frames in failure, etc.). The results indicated distinct behavior patterns for at least three different types of scenarios.

- Commercial and business scenarios: shopping centers, restaurants, hotels, entertainment venues, etc.

- Public scenarios: schools, university campuses, cultural venues, museums, etc.

- Residential scenarios: both residential wireless access and shared agreement access

Therefore, it was decided to carry out the study in three such real Wi-Fi scenarios:

- The Custom House Quay (CHQ) building (https://chq.ie): to cover both business and commercial scenarios. This building, situated within the heart of Dublin, holds different food and shopping spaces.

- Dublin Institute of Technology (http://www. dit.ie/): to cover public (e.g., educational campus) scenarios.

- The Gasworks Area: to cover residential scenarios.

Subjective and objective measurements were required to validate the methodology. Objective Wi-Fi data were collected through OptiWi-fi net- 


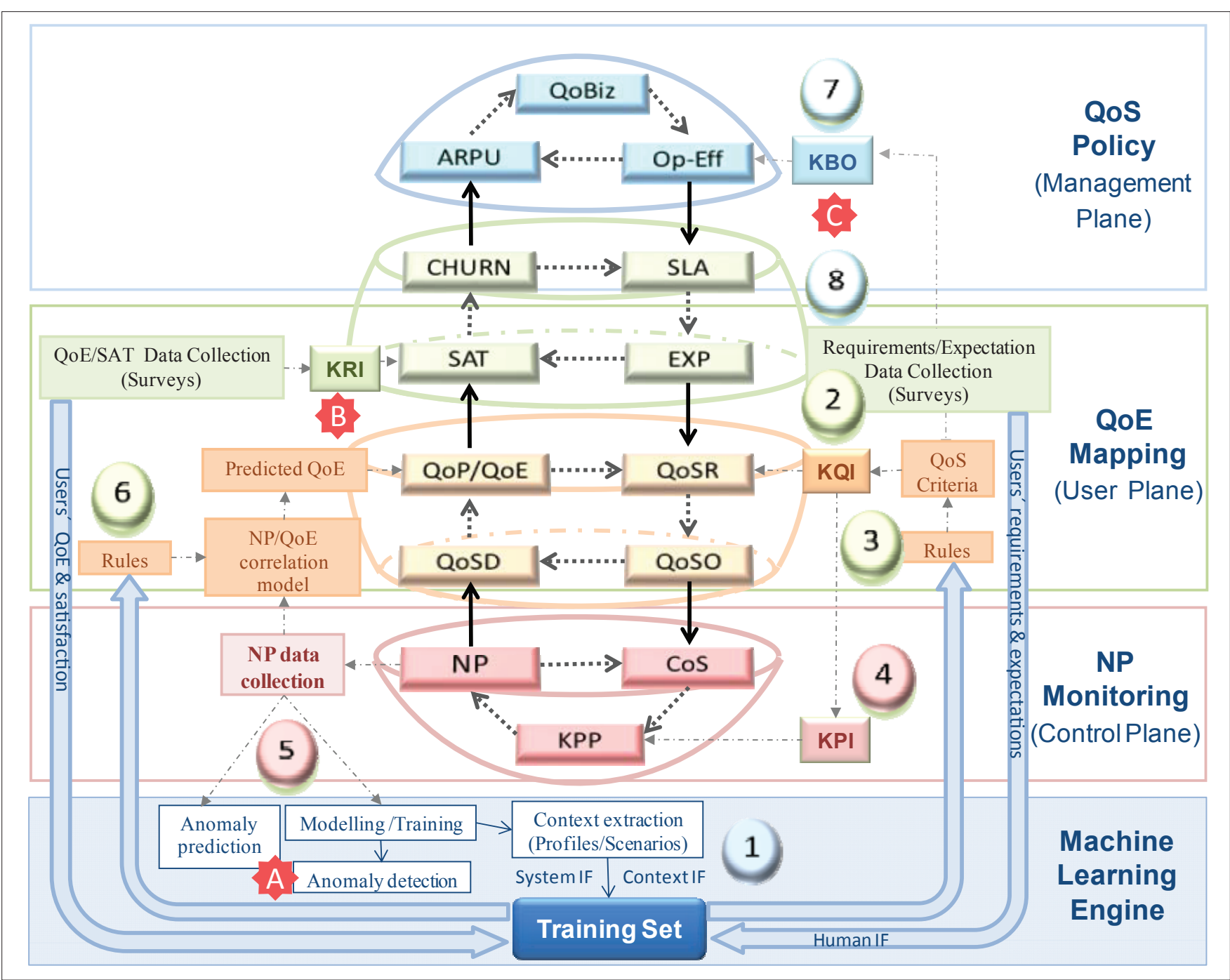

FIGURE 5. Proposed methodology for machine learning in 5G.

work probes described in [14]: NP data, location data, mobility patterns, number of APs, number of clients, connection time, types of device, and other information, according to [15], were captured by the probes. Subjective data were gathered through user surveys: personal information (gender, age, occupancy, Internet expertise) together with the data related to the "Wi-Fi experience," both about the user's requirements/ expectations and QoE satisfaction with the service, were collected in the same survey.

At least 50 participants completed the survey in each of the scenarios during the test field, carried out from January to June 2018.

While the surveying process was carried out in a one-week period for each venue, due to the complexity of the procedure, the network probes were deployed for a month in each of the venues to capture the required information about context and system influence factors.

\section{QOS CRITERIA AND KQIS}

The data collected from the probes and the surveys shed light on the important differences between the users' influence factors in the different scenarios. In addition, the results of the surveys about the user's requirements for each of the scenarios also showed that the relevant QoS criteria and KQIs differ from one scenario to another (Fig. 6).

Although the use of ML to automatically update the KQIs has not been tested yet, it has been shown to be essential given the number and changing nature of the influence factors.

\section{Control Plane:}

Anomaly Detector and PREDictor The data collected from the probes have been used not only to learn about the users' behavior and extract the context information, but also to detect anomalies and enhance the channel selection process through unsupervised ML.

\section{User Plane: Supervised ML}

Based on previous research studies [8], inductive supervised learning has been employed for the NP-QoE correlation model.

The results of the surveys concerning user's QoE and satisfaction were fed into the model to infer the rules to automatically predict the QoE based on NP and the influence factors. In future stages of the study, ML techniques will be also implemented to enhance the satisfaction model (CSAT) described in [12]. 


\section{Results: CoRrective Actions to ENHANCE QOE}

Although the validation of the methodology is still at a premature stage, the case study has revealed that the proposed methodology can be useful to deploy the QoS management model and enhance the user's QoE. In fact, the results have indicated several corrective actions that could be implemented through $\mathrm{ML}$ in the scenarios studied here.

- Commercial scenario: One of the most relevant KQIs in this scenario (streaming video/ audio application performance) is affected by NP problems (continuous disruptions of the service). Corrective action: Analysis of NP data for AP capacity and the use of ML to enhance the channel selection mechanism.

- Campus Scenario: Some client-association problems have been found due to poor AP configuration. Furthermore, the students were dissatisfied with one of the KQIs (the ease of login) so the procedures around the login procedure should be revised. Corrective actions: Customize AP performance through ML techniques and revise login procedures according to the learned rules.

- Residential scenario: Lower cost and higher network speed have been two of the key user requirements in this scenario. Corrective actions: Customize residential Wi-Fi APs for optimal throughput through $\mathrm{ML}$ and enhance the business model using supervised ML through the survey's observation set.

\section{CONCLUSIONS}

In this article a methodology to implement a global QoS management model for the next generation wireless ecosystem leveraging big data and $\mathrm{ML}$ techniques has been presented.

Taking into account various international standards, the QoE-centric approach makes use of supervised $M L$ techniques in order to identify the KQIs relevant to the users. Unsupervised ML mechanisms are proposed for the identification of the users' influence factors, network performance anomalies, fault detection, and channel selection enhancement. The proposed approach links the NP and QoE via inductive ML techniques and identifies intervention points where corrective actions are required.

Although the definition of the methodology and the validation of the approach are still at an early stage, the results of the case study, carried out in a number of different Wi-Fi scenarios, reveal that the methodology may aid enhanced QoE in next generation wireless environments. In addition, some recent studies suggest that $\mathrm{ML}$ techniques may be applied for customer retention to enhance the QoBiz, and these will also be analyzed at future research stages of this proposal.

\section{REFERENCES}

[1] ITU-T Rec. Y.3100, "Terms and Definitions for IMT-2020 Network," 2017.

[2] ITU-T Rec. Y.3170, "Requirements of Machine Learning Based OoS Assurance for IMT-2020 Network," 2018.

[3] ITU-T Focus Group on Machine Learning for Future Networks Including 5G, 2017; https://www.itu.int/en/ITU-T/ focusgroups $/ \mathrm{ml} 5 \mathrm{~g} /$

[4] ITU-T Rec. E.804, "Quality of Service Aspects for Popular Services in Mobile Networks," 2014.

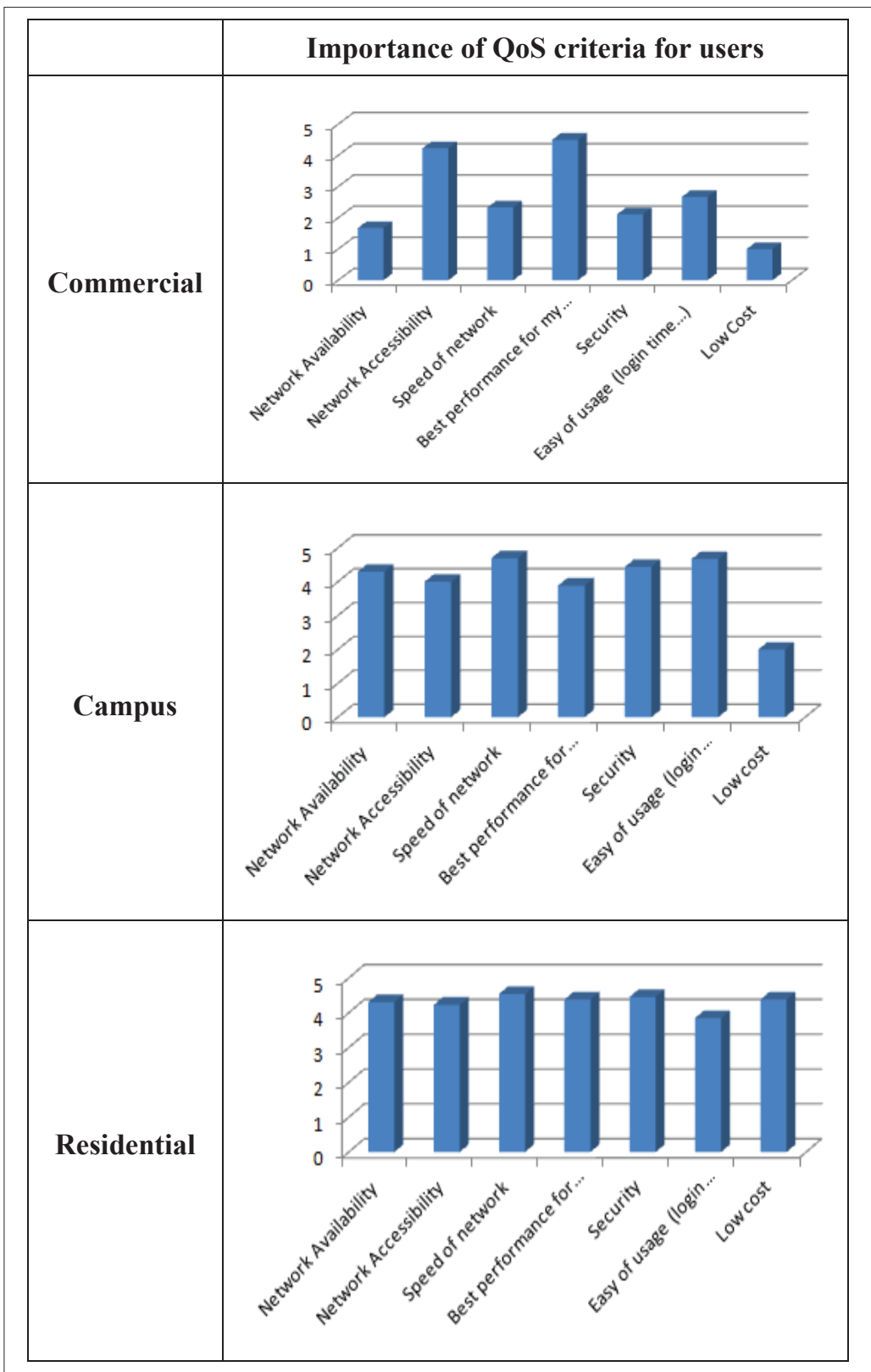

FIGURE 6. KQI relevance in different scenarios.

[5] ITU-T Draft New Rec. G.QoE-5G, "QoE Factors for New Services in 5G Network," 2018.

[6] ITU-T Rec. G.1000, "Communications Quality of Service: A Framework and Definitions," 2001.

[7] A. W. Yusuf-Asaju, Z. M. Dahalin, and A. Ta'a, "Framework for Modelling Mobile Network Quality of Experience Through Big Data Analytics Approach," J. Info. and Commun. Technology, vol. 17, 2018, pp. 79-113.

[8] S. Aroussi and A. Mellouk, "Survey on Machine Learning-Based QoE-QoS Correlation Models," Proc. Int'l. Conf. Computing, Management and Telecommun., Vietnam, 2014, pp. 200-04.

[9] C. Jiang et al., "Machine Learning Paradigms for Next-Generation Wireless Networks," IEEE Wireless Commun., vol. 24, 2017, pp. 98-105.

[10] ITU-T Draft New Recommendation Y.IMT-2020-qos-fa, "QoS Functional Architecture for the IMT-2020 Network," 2019.

[11] ITU-T Rec. E.802, "Framework and Methodologies for the Determination and Application of QoS Parameters," Geneva, Switzerland, 2007.

[12] E. Ibarrola et al., "A New Global Quality of Service Model: QoXphere," IEEE Commun. Mag., vol. 52, 2014, pp. 193-99.

[13] ITU-T Rec. E.419, "Business Oriented Key Performance Indicators for Management of Networks and Services," Geneva, Switzerland, 2006. 
[14] M. Davis, "A Wireless Traffic Probe for Radio Resource Management and QoS Provisioning in IEEE 802.11 WLANs," Proc. 7th ACM Int'l. Symp. Modeling, Analysis and Simulation of Wireless and Mobile Systems, Venice, Italy, 2004, pp. 234-43.

[15] M. Davis and T. Raimondi, "A Novel Framework for Radio Resource Management in IEEE 802.11 Wireless LANs,," presented at the 3rd Int'l. Symp. Modeling and Optimization in Mobile, Ad Hoc, and Wireless Networks, 2005, pp. 139-47.

\section{BIOGRAPHIES}

Eva IBARRola (eva.ibarrola@ehu.eus) received her Ph.D. degree in telecommunications engineering in 2010 from the University of the Basque Country (UPV/EHU), Spain. She was honored with the Best Thesis Award in Management, Economy and Telecommunications Regulation for her work in the area of user-centric QoS management models. She is currently an associate professor in the Faculty of Engineering in Bilbao in the area of telecommunications networks and services. Prior to joining the University of the Basque Country in January 2000, she worked at the National Monitoring and Operation Center of Telefónica in Madrid. She has been participating in different R\&D projects and cooperating on different standardization bodies in the area of user-centric QoS management models and frameworks.

MARK DAVIS (mark.davis@tudublin.ie) received his B.E. (1986) M.Eng.Sc. (1998), and Ph.D. (1992) degrees from University College Dublin in the fields of electronic and communications engineering. Since 1994 he has lectured at Technological University Dublin (formerly Dublin Institute of Technology) in the area of wireless communications and is currently an assistant head of school in the School of Electrical and Electronic Engineering. He is also the director of the Communications Network Research Institute (CNRI) where his research interests include QoS provisioning for Wi-Fi networks and the application of machine learning techniques to the performance monitoring and management of wireless networks. He also holds several patents in the area of Wi-Fi networks and is a co-founder of a spin-out company, OptiWi-fi.

CAMILLE VOISIN (camille.voisin@optiwifi.com) received his M.S degree in computer science and telecommunications from the University ENSIMAG-telecom in Grenoble, France, in 2002 He joined Dell, where he became IT director, managing Dell's global ERP implementation of "Order to Cash" on Oracle eBusiness Suite, a unique and complex program to deploy one of the world's largest instances of Oracle applications. In 2014, He then joined OptiWi-fi as chief technology officer to develop and commercialize technologies to monitor, manage, and optimize Wi-Fi networks.

CIARA ClOSE (ciara.close@ucdconnect.ie) received her B.E. in electronic engineering from the University of Calfornia Davis (UCD) in 2004. She carried out research into holographic materials for data storage, earning her P.h.D in optical engineering in 2012. She worked with OptiWi-fi in research and product development roles, collaborating with customers and partner organizations. She is currently completing an M.B.A. with UCD and New York University focusing on technology innovation and sustainability.

LEIRE CRISTOBO (leire.cristobo@ehu.eus) received B.S. and M.S degrees in telecommunications engineering from the University of the Basque Country in 2004. She has nine years of communications engineering experience and is currently head of the IT Department at ST3 Elkartea. Since 2007 she has been an associate professor at the University of the Basque Country, where she is currently a Ph.D. candidate. She conducts research in the areas of QoE and quality of business (QoBiz) management processes. 